\title{
Efficacy of Pulse Therapy of Oral Fluconazole in the Treatment of Seborrheic Dermatitis
}

\author{
Khondker L1, Wahab MA2, Khan MSI3.
}

\begin{abstract}
An interventional study was carried out for a period of total two years in the department of Dermatology and Venereology, Bangabandhu Sheikh Mujib Medical University (BSMMU), Dhaka, Bangladesh. Total sixty five patients of seborrheic dermatitis were selected and were treated with oral fluconazole 150mg in a single dose per week for 4 weeks. Follow up were done at the end of 4 th week and 8th week. Among the patients, 31-45 years age group was highest $44 \%$, highest $54 \%$ males and 21 (32\%) had positive family history and 44 (68\%) had negative family history of seborrheic dermatitis. Among the sixty five patients, mild form was $54 \%$, moderate was $22 \%$ and severe was $12 \%$. Highest patients of seborrheic dermatitis $51 \%$ had the duration of lesion 1 to 3 years and next $38 \%$ had the duration 4 to 6 years. Regarding site of lesions, maximum patients of seborrheic dermatitis $92 \%$ had involvement of scalp; next $46 \%$ had involvement in the eyebrow. The study showed that the response was very good in 31.5 of cases, good response was found in $24.5 \%$ of cases, fair in $26 \%$ of cases and poor response was observed in $18.5 \%$ of cases. The study showed that $83 \%$ of study population was seen without clinical side-effect and only $17 \%$ were seen with side-effect(anorexia and dydpepsia) and it was showed that very good improvement 35\% observed on the 1st follow up visit at the fourth week, $30 \%$ had good, 15\% fair and poor improvement 20\% respectively. On the 2 nd follow up visit at the end of eight week, very good improvement was 39\% cases; good, fair and poor improvement was $26 \%, 20 \%$ and $17 \%$ respectively. The results of this study indicate that fluconazole provides benefit for the therapy of seborrheic dermatitis. However, larger studies using different dosages and durations of therapy, fluconazole may provide a rationale for systemic use of fluconazole in seborrheic dermatitis.
\end{abstract}

\section{Introduction}

Seborrheic dermatitis is a common chronic superficial papulo-squamous dermatosis that is often associated with

1. Corresponding Author: Dr. Lubna Khondker, MBBS, MPH,DDV,MCPS, FCPS Assistant Professor, Department of Dermatology and Venereology Bangabandhu Sheikh Mujib Medical University E-mail: lubna_derma@yahoo.com.

2. Lt. Col. (Retd) Dr Md Abdul Wahab Professor, Department of Dermatology and Venereology Bangabandhu Sheikh Mujib Medical University

3. Lt. Col. Dr. Md Shirajul Islam Khan, MBBS, DDV, MCPS Graded Specialist in Dermatology and Venereology Combined Military Hospital, Dhaka increased sebum production (seborrhea) of the scalp and the sebaceous follicle-rich areas of the face and trunk. Seborrheic dermatitis, also known as Seborrheic eczema, occurring in $2 \%$ to $5 \%$ of the population ${ }^{1}$. The affected skin is erythematous and covered with yellow-brown scales and crusts. The disease varies from mild to severe; including psoriasiform patterns and erythroderma ${ }^{2}$. Patients with human immunodeficiency.

virus (HIV) infection have an increased risk of seborrheic dermatitis $^{3}$. Consequently, it is included in the spectrum of premonitory lesions and should be carefully evaluated in high-risk patients ${ }^{3}$. The skin commensal yeasts Malassezia is known to cause the disease. Correlation of severity of the disease with the number of yeasts and decrease in the number of Malassezia after treatment seem to support that this may be caused by Malassezia yeast ${ }^{4}$. Although there is no definitive care, topical corticosteroids, tar, sulfur, pyrithione sulfide, ciclopiroxolamine, terbinafine, butenafine, azoles such an ketoconazole, bifonazole, itraconazole, metronidazole, fluconazole; tacrolmus, pimecrolimus, lithium succinate and cinnamic acid may be used. However, the relapse rate is high and the duration of remission generally short. In severe disease, systemic antifungals that are used in the treatment of seborrheic dermatitis are ketoconazole, itraconazole and terbinafine ${ }^{5}$. Fluconazole is distinguished from the other azole medications by its water solubility and good cerebrospinal fluid penetration. So an excellent bioavailability can be gained by oral administration. Drug interaction are also less common because fluconazole has the least effect on hepatic microsomal enzymes. For this reason and also for better gastrointestinal tolerance, fluconazole has the widest therapeutic index of the azole, permitting more aggressive dosing in a variety of fungal infections ${ }^{6}$. Since seborrheic dermatitis is a relapsing condition, use of topical agents may not be suitable on a long-term basis and oral treatment is preferred by patients who are refractory to topical treatment, relapse frequently, or have disease that affects large areas ${ }^{4}$.

\section{Materials and Methods}

The interventional study was carried out for a period of total two years from January 2009 to December 2010 in the department of Dermatology and Venereology, Bangabandhu Sheikh Mujib Medical University (BSMMU), Dhaka in Bangladesh. Total sixty five patients of seborrheic dermatitis were selected considering exclusion criteria like patient with known hypersensitivity to any ingredients of the fluconazole, pregnancy/lactation, impaired hepatic function, impaired renal function and severe systemic illness. The inclusion criteria of patient selection were both 
male and female patient of age 15-60, patient willing to give consent to take part in the study, patient expected to be available for the duration of study and able to comply with the study visit and patient received no topical treatment for 2 weeks prior to the study and no systemic antifungal intake. Purposive type non-probability sampling technique was followed in this study. After collection of data, these were screened by checking consistency, edited and were finally analyzed by software SPSS (Statistical Package for Social Science) method.

\section{Procedure of Treatment}

The patient of seborrheic dermatitis was identified first. The diagnosis was made on the clinical basis by assessing morphology of lesions, age of onset and their distribution sites. To reach a clinical diagnosis detailed history and thorough physical examination done. Then clinical conditions of the patient were recorded by us. Skin biopsy was also performed for histopathological examination, along with hematological and biochemical profile, like blood for total count, differential count, ESR, platelet count, bleeeding time, clotting time, random blood sugar, serum for cholesterol and triglyceride level, serum for ALT and serum creatinine level. Then verbal and written concent was taken from the selected patient and were interviewed by asking questions. Finally, all patients with seborrheic dermatitis were treated by oral fluconazole $150 \mathrm{mg}$ in a single dose per week for 4 weeks. The cases were divided as mild from (dandruff, red and flaky skin),moderate form (thick, oily and yellow scales) and severe from (generalized exfoliative erythroderma) and patient's subjective assessment of pruritus and burning sensation were evaluated before and after treatment. A final medical assessment of efficacy is made at the end of the treatment period using a three point scale (categories: very good-more than $75 \%$ clearing, good- $50-75 \%$ clearing, fair- $25-50 \%$ clearing, poor less than $25 \%$ clearing) and the assessment result is recorded and analyzed to prepare the final result. Follow up were done at the end of 4th week and 8th week.

\section{Results}

The study was carried out for a period of total two years from January 2009 to December 2010 in the department of Dermatology and Venereology, Bangabandhu Sheikh Mujib Medical University (BSMMU), Dhaka in Bangladesh. Total sixty five patients of seborrheic dermatitis were selected. Among them, 31-45 years age group was 44\%, 15-30 years was $38 \%$ and $46-60$ years age group was $12 \%$, regarding sex,35 (54\%) males and $30(46 \%)$ females between $15-60$ years aged patients with seborrheic dermatitis and regarding family history, $21(32 \%)$ had positive family history and $44(68 \%)$ had negative family history of seborrheic dermatitis (Table I). Regarding occupation among the patients, $50 \%$ were outdoor worker, $38 \%$ were involved in indoor service and rest $12 \%$ involved in other occupation (Figure I). Among the sixty five patients of seborrheic dermatitis in table II, mild form was $54 \%$, moderate was
$22 \%$ and severe was $12 \%$. Regarding duration of lesions, highest patients of seborrheic dermatitis $51 \%$ had the duration 1 to 3 years and next $38 \%$ had the duration 4 to 6 years. Regarding site of lesions, maximum patients of seborrheic dermatitis $92 \%$ had involvement of scalp, next $46 \%$ had involvement in the eyebrow, $41 \%$ had involvement in back etc.

Table I: Distribution of the patient by epidemiological profile $(n=65)$.

\begin{tabular}{|lc|}
\hline Epidemiological profile & Frequency \\
Age(in years) & \\
$15-30$ & $25(38 \%)$ \\
$31-45$ & $28(44 \%)$ \\
$46-60$ & $12(18 \%)$ \\
Sex & \\
Male & $35(54 \%)$ \\
Female & $30(46 \%)$ \\
Family History & $21(32 \%)$ \\
Positive & $44(68 \%)$ \\
Negative & $\square$ Worker (outdoor \\
\hline & $\square$ Service (indoor) \\
\hline & $\square$ Others \\
\hline
\end{tabular}

Figure I: Distribution of the patient by occupation.

Table II: Distribution of the patient by different form of disease, duration of lesions and site of lesions $(n=65)$.

\begin{tabular}{|lc|}
\hline Parameters & Frequency \\
Different forms & \\
\hline Mild & $35(54 \%)$ \\
Moderate & $22(34 \%)$ \\
Severe & $8(12 \%)$ \\
Duration of lesion & \\
Less than 1 year & $5(8 \%)$ \\
1 to 3 years & $33(51 \%)$ \\
4 to 6 years & $25(38 \%)$ \\
More than 6 years & $2(3 \%)$ \\
Site of lesion (multiple response may exceed hundred) \\
Scalp & $60(92 \%)$ \\
Forehead & $20(31 \%)$ \\
Nasolabialfold & $25(38 \%)$ \\
Eyebrow & $30(46 \%)$ \\
Ear & $22(34 \%)$ \\
Back & $27(41 \%)$ \\
Chin & $25(38 \%)$ \\
Intermammary & $19(29 \%)$ \\
\hline
\end{tabular}


Table III: showed that $43 \%$ patients of seborrheic dermatitis had very good response, $28 \%$ good response, $20 \%$ fair response and $9 \%$ poor response in mild form of seborrheic dermatitis. Patients with moderate form show that $23 \%$ had good response, $27 \%$ good response, $36.5 \%$ fair response and $3 \%$ poor response. And patients with severe form showed no very good response and good response but $2 \%$ fair response and $6 \%$ poor response in treating patients of seborrheic dermatitis. In total, the response was very good in 31.5 of cases, good response was found in $24.5 \%$ of cases, fair in $26 \%$ of cases and poor response was observed in $18.5 \%$ of cases.

Table III: Distribution of the patient by response of therapy at the end of the study $(n=65)$.

\begin{tabular}{|lcccc|}
\hline Forms of seborrheic dermatitis & Very good & Good & Fair & Poor \\
\hline Mild $(\mathrm{n}=35)$ & $43 \%$ & $28 \%$ & $20 \%$ & $9 \%$ \\
Moderate(n=22) & $23 \%$ & $27 \%$ & $36.5 \%$ & $3 \%$ \\
Severe(n=8) & $0 \%$ & $0 \%$ & $2 \%$ & $6 \%$ \\
Total $(\mathrm{n}=50)$ & $31 \%$ & $24.5 \%$ & $26 \%$ & $18.5 \%$ \\
\hline
\end{tabular}

Table IV: showed that $83 \%$ of study population were seen without clinical side-effect and only $17 \%$ were seen with side-effect like anorexia and dyspepsia which was not significant. Nobody had discontinued therapy for sideeffects and not required any additional treatment for sideeffect.

Table-IV: Distribution of the patients by side effect.

\begin{tabular}{|lcc|}
\hline Safety & Number & Percentage \\
\hline With out side-effect & 54 & $83 \%$ \\
With side-effect & 11 & $17 \%$ \\
\hline
\end{tabular}

Figure II showed that very good improvement $35 \%$ observed on the 1st follow up visit at the fourth week, $30 \%$ had good, fair $15 \%$ and poor improvement $20 \%$ respectively. On the 2 nd follow up visit at the end of eight week, very good improvement was $39 \%$ cases, good, fair and poor improvement was $26 \%, 20 \%$ and $17 \%$ respectively.

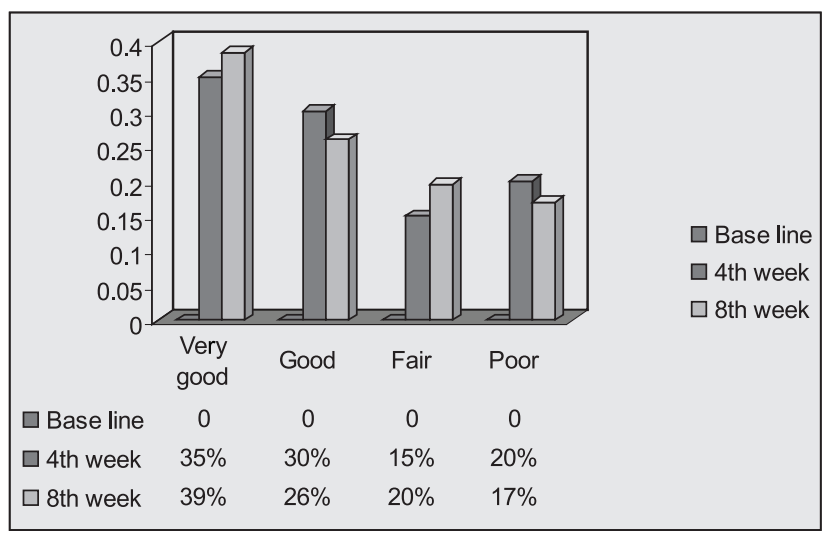

Figure II: Distribution of the patient by follow up after fluconazole therapy.

\section{Discussion}

The study was carried out for a period of total two years from January 2009 to December 2010 in the department of Dermatology and Venereology, Bangabandhu Sheikh Mujib Medical University (BSMMU), Dhaka, Bangladesh. Total sixty five patients of seborrheic dermatitis were selected. In this study, regarding occupation among the patients, 50\% were outdoor worker, $38 \%$ were involved in indoor service and rest $12 \%$ involved in other occupation. Fifty percent of total patients are outdoor worker used to exposure in sunlight and hot humid climate for their nature of occupation. This reflects the precipitating factor of seborrheic dermatitis.

The study showed that $43 \%$ patients of seborrheic dermatitis had very good response, $28 \%$ good response, $20 \%$ fair response and $9 \%$ poor response in mild form of seborrheic dermatitis. Patients with moderate form show that $23 \%$ had good response, $27 \%$ good response, $36.5 \%$ fair response and $3 \%$ poor response. And patients with severe form showed no very good response and good response but $2 \%$ fair response and $6 \%$ poor response in treating patients of seborrheic dermatitis. In total, the response was very good in 31.5 of cases, good response was found in $24.5 \%$ of cases, fair in $26 \%$ of cases and poor response was observed in $18.5 \%$ of cases.

The study by Robert, Schwartz and Christopher showed that short-term fluconazole treatment may improve the clinical features of mild-to-moderate seborrheic dermatitis. Several topical and systemic antifungals had been used in the treatment of seborrheic dermatitis with varying success rates $^{7}$. The efficacy of oral antifungals was attributable to their antifungal and/or anti-inflammatory effects by Gupta, Nicol and Batra $^{8}$. Systemic use of ketoconazole, itraconazole, and terbinafine has been associated with good clinical response in the treatment of seborrheic dermatitis. However, the efficacy of oral fluconazole which was highly effective against a wide spectrum of dermatophytes and yeasts, had been tried in seborrheic dermatitis and showed marked improvedment ${ }^{9,10}$. In one study, oral fluconazole $150 \mathrm{mg}$ as a single dose per week was given for 4 weeks. This dosage was chosen because it has been used effectively and safely in the treatment of tinea versicolor. Furthermore, the long-term safety of fluconazole has been established in patients with onychomycosis receiving 150-300mg weekly dosages for 6-12 months by Coldiron $\mathrm{B}^{11}$. During therapy with fluconazole, the drug concentration in skin reaches ten times that in plasma and the drug is eliminated from the skin very slowly. This allows once-weekly administration, improving patient compliance and reducing costs compared with daily administration, seen by Montero-Gei and Perera $^{12}$.

The study showed that very good improvement 35\% observed on the 1st follow up visit at the fourth week, 30\% had good, fair $15 \%$ and poor improvement $20 \%$ respectively. On the 2 nd follow up visit at the end of eight week, very good improvement was $39 \%$ cases, good, fair and poor improvement was $26 \%, 20 \%$ and $17 \%$ 
respectively. Since seborrheic dermatitis is a relapsing condition, use of topical agents may be unsuitable on a longterm basis and oral treatment is preferred by patients who are refractory to topical treatment, relapse frequently, or have disease that affects large areas. The safety profile of fluconazole when used on a long term basis, its efficacy against yeasts, and the cost effectiveness of pulse therapy make fluconazole a therapeutic option in 'recalcitrant cases' of seborrheic dermatitis ${ }^{8}$.

It was showed that $83 \%$ of study population was seen without clinical side-effect and only $17 \%$ were seen with side-effect like anorexia and dyspepsia, which was not significant. Nobody had discontinued therapy for sideeffects and not required any additional treatment for sideeffect.

Our study had several limitations. First, no fungal culture was performed and the clinical outcome could not therefore be correlated with Malassezia yeast colonization. Consequently, a possible anti-inflammatory effect of fluconazole could not be evaluated. In addition, the selfremitting course of the disease, the number of patients and the duration of treatment in this study may have been insufficient to evaluate drug-related improvement. The current study was an attempt to develop a short, costeffective, convenient, and safe treatment protocol, which is strongly needed for seborrheic dermatitis.

The results of this study indicate that fluconazole provides benefit for the patients of seborrheic dermatitis. However, larger studies using different dosages and durations of therapy may provide a rationale for systemic use of fluconazole in seborrheic dermatitis.

\section{References}

1. Freedberg IM, Eisen AZ, Wolff K, Austen KF, Goldsmith LA and Katz SI. Fitzpatrick's Dermatology in General Medicine. 6th edition. New York: The McGraw-Hill Companies. 2003;1198-1204.

2. James WD, Berger TG and Elston D. Andrews' Diseases of the skin- Clinical Dermatology. 10th edition. USA: Saunders Elsevier. 2006;191-192.

3. Gupta AK and Bluhm R. Seborrheic dermatitis. Journal of European Academy of Dermatology and Venereology. 2004;18:13-26.

4. Dunic I, Vesic S, Jevtovic DJ. Oral candidiasis and seborrheic dermatitis in HIV infected patients on highly active antiretroviral therapy. HIV Med. 2004;5:50-54.

5. Bergbrant IM. Seborrhoeic dermatitis and Pityrosporum ovale: cultural, immunologic and clinical studies. Acta Derm Venereol. 1991;167:10-36.

6. Hay RJ, Graham-Brown RA."Dandruff and seborrhoeic dermatitis: causes and management". Clinical and Experimental Dermatology. 1997;22:3-6.

7. Robert A, Schwartz RA, Christopher AJ.Treatment of Seborrheic dermatitis. American Academy of Family Physicians. 2006;61:2703-10.

8. Gupta AK, Nicol K, Batra R. Seborrheic dermatitis. Dermatol Clin. 2004;21:401-412.

9. Zisova LG. Infantile seborrheic dermatitis Cutis. May 2006;77:297-300.

10. Bodyey GP. "Seborrheic dermatitis: an overview". American Family Physician. 2006;74:125-30.

11. Coldiron B. "Seborrheic dermatitis". American Family Physician. 1995;52:149-55, 159-60.

12. (Montero-Gei F, Perera A, Nowicki R. "Modern management of dandruff "(in Polish). Polski Merkuriusz Lekarski. 2006;20:121-4. 